\title{
Technical note: The equivalency of sodium results in cheese digested by either dry ashing or microwave-accelerated digestion
}

\author{
T. C. Schoenfuss, ${ }^{\star 1}$ Z. P. Metz, $†$ A. E. Pataky, ${ }^{\star}$ and H. L. Schoenfuss $\ddagger$ \\ *Department of Food Science and Nutrition, University of Minnesota, Saint Paul 55108 \\ †Chemical Engineering and Materials Science, University of Minnesota, Minneapolis 55455 \\ ¥Aquatic Toxicology Laboratory, St. Cloud State University, St. Cloud, MN 56301
}

\begin{abstract}
Analysis of dairy products for minerals such as sodium requires mineralization of the sample, which is typically done by either dry ashing or atmospheric wet ashing; both methods are time consuming and wet ashing requires the repeated handling of hot acid. A rapid method using microwave-accelerated acid digestion before atomic absorption spectrometry to measure sodium was compared with dry ashing in 138 samples of blue cheese (in duplicate) that varied in sodium content and age. Linear regression of the results obtained within different cheese salting treatments and sampling locations over time showed that the methods were equivalent in terms of linearity and the slope of the line. A consistent bias was observed, with lower sodium concentrations being quantified during atomic absorption spectrometry for the microwave-digested samples. Evaluation of this difference by the 2 one-sided test (TOST) procedure showed that the confidence intervals of the percentage difference between the methods fell within the predetermined acceptable percentage difference. We conclude that this rapid microwave digestion procedure of blue cheese yielded equivalent results to dry ashing.
\end{abstract}

Key words: atomic absorption, sodium in cheese, microwave-accelerated reaction, digestion

\section{Technical Note}

Mineral analysis of food products can be a lengthy and time-consuming process. Organic compounds must first be destroyed by wet or dry ashing before analysis by a method such as atomic absorption spectrometry (AAS) or inductively coupled plasma-optical emission spectrometry (ICP-OES). For measurement of minerals such as sodium in cheese, wet and dry ashing are used (ISO-IDF, 2007). With wet ashing at atmospheric pressure, the sample is boiled in concentrated

Received August 26, 2013.

Accepted November 2, 2013.

${ }^{1}$ Corresponding author: tschoenf@umn.edu nitric acid in a flask with a watch glass cover, for 2 h. Dry ashing involves acid soaking of crucibles to remove residual minerals, lighting the cheese weighed into the crucible on fire to carbonize it, followed by ashing in a muffle furnace at $550^{\circ} \mathrm{C}$ for at least $6 \mathrm{~h}$. As a result, the sample preparation steps for both of these methods consume at least $1 \mathrm{~d}$ before analysis by AAS or ICP-OES. These methods also expose the operator to strong acids and high temperatures, thus invoking safety concerns. Microwave-accelerated wet digestion (MAD) is a technique where sample and acid are placed in a polytetrafluoroethylene (PTFE) reaction vessel and heated to digest the sample. This is a much faster and self-contained means of digestion and could greatly improve the speed for analyzing products (such as cheese for sodium), reduce the use of concentrated acid, and reduce exposure to hazards for the operator. The International Dairy Federation/ International Organization for Standardization method IDF119:2007/ISO 8070:2007 (IDF-ISO, 2007) describes mineral quantification by AAS in dairy products with several digestion options, one being microwave digestion. Pressurized MAD is described in general in this method, but is unspecific for conditions required except in specifying microwave ashing at $150^{\circ} \mathrm{C}$ for at least $3 \mathrm{~h}$. An AOAC International official method exists for microwave digestion of fortified food products followed by ICP-OES, but cheese was not included and hydrochloric acid is used instead of nitric acid (AOAC International, 2011). One collaborative interlaboratory study included MAD of cheese before sodium analysis by AAS, and microwave digestion was determined to produce accurate results (Julshamn et al., 2005). Multiple microwave oven types were used in the aforementioned study, and digestion temperature, wattage, sample size, and acid amount were not controlled. One cheese was sampled by all laboratories evaluating the method, so variations in sodium concentration or matrix were not evaluated nor were comparisons made to a standard sample preparation procedure.

A microwave-accelerated reaction system produced by CEM Corp. (Matthews, NC) has the ability to monitor the temperature of each vessel (24 per carousel) as they 
rotate in a carousel and adjust the power to maintain a constant average temperature for all vessels throughout the hold time. We compared this system of MAD to standard dry ashing in 138 samples of blue cheese, in duplicate, with sodium concentrations ranging from approximately 780 to $1,300 \mathrm{mg} / 100 \mathrm{~g}$ of cheese. The effect of variation in sodium concentration and cheese chemistry due to cheese aging on the equivalency of the methods was evaluated.
Surface-salted blue cheese wheels (weighing approximate $3 \mathrm{~kg}$ each) were sampled at 2 locations per wheel at monthly intervals during aging to monitor sodium migration. Cheeses were manufactured as part of a sodium reduction study and the treatments were (1) control cheese, (2) $25 \%$ reduced sodium, and (3) $25 \%$ reduced sodium with $\mathrm{KCl}$. The gross composition at initial sampling is shown in Table 1. The amount of $\mathrm{NaCl}(\mathrm{g})$ applied per wheel was calculated as $0.035 \times$
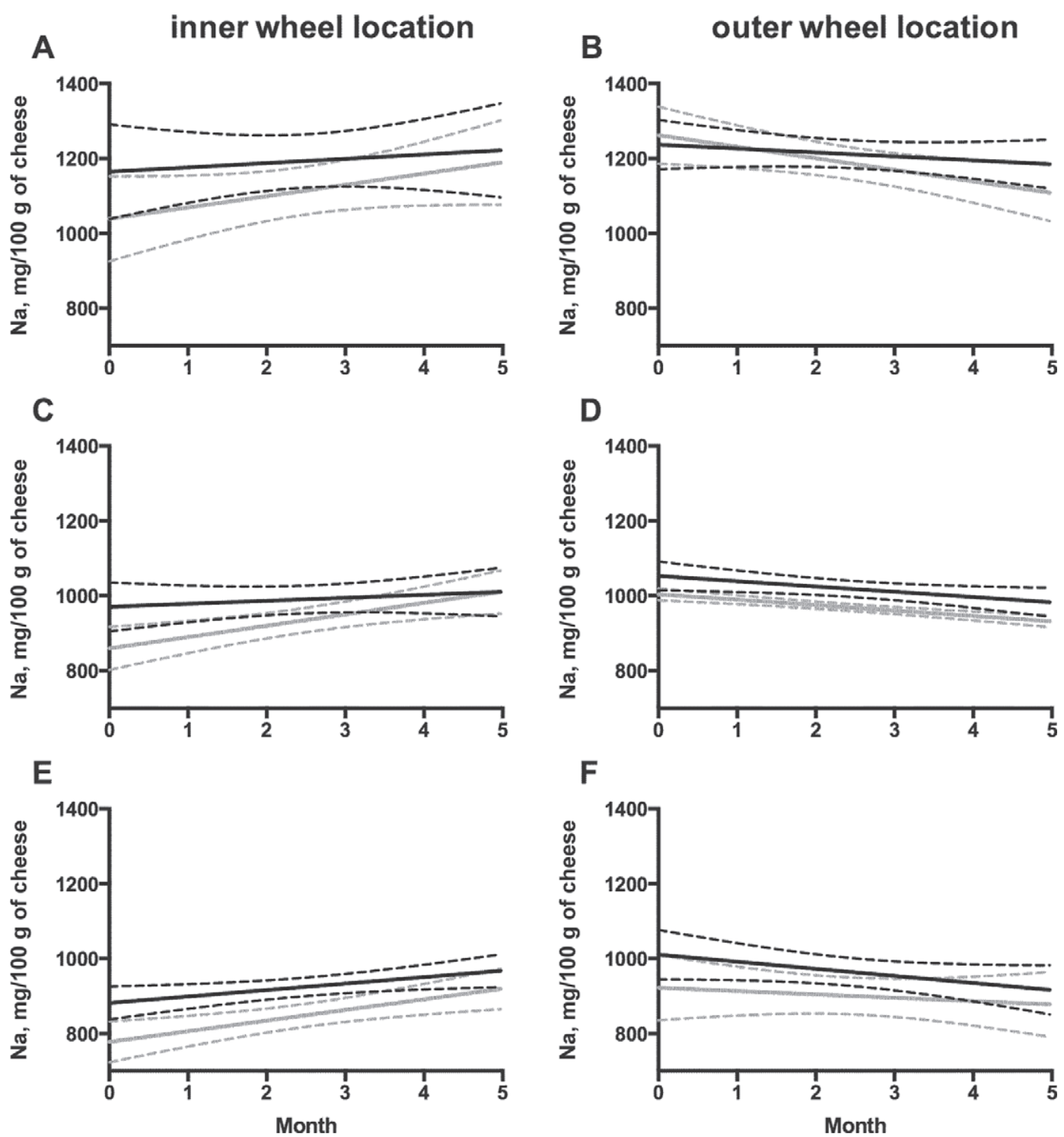

Figure 1. Sodium measured over 5 mo of ripening for blue cheese samples digested by either dry ashing (black solid lines) or microwave digestion (gray solid lines); dashed lines indicate $95 \%$ confidence intervals. Panels A, C, and E are measurements obtained from sampling the inner portion of the wheel of blue cheese for control, reduced sodium, and reduced sodium with $\mathrm{KCl}$, respectively. Inner location was cut from the core of the wheel, $>5.1 \mathrm{~cm}$ from the surface, $>1.26 \mathrm{~cm}$ from the top. Panels B, D, and F are for cheese samples from the respective treatments sampled at an outer wheel location. Outer location sampled from between 0.63 and $4.4 \mathrm{~cm}$ from the surface of the wheel. Treatments consisted of different salting applications. The amount of $\mathrm{NaCl}(\mathrm{g})$ applied per wheel was calculated as $0.035 \times$ unsalted wheel weight $(\mathrm{g})$ for the control treatment, and as $0.0245 \times$ unsalted wheel weight $(\mathrm{g})$ for both reduced sodium treatments $(\mathrm{KCl}$ was added with the $\mathrm{NaCl}$ to the treatment with $\mathrm{KCl}$ at $0.0134 \times$ unsalted wheel weight, $\mathrm{g})$. 


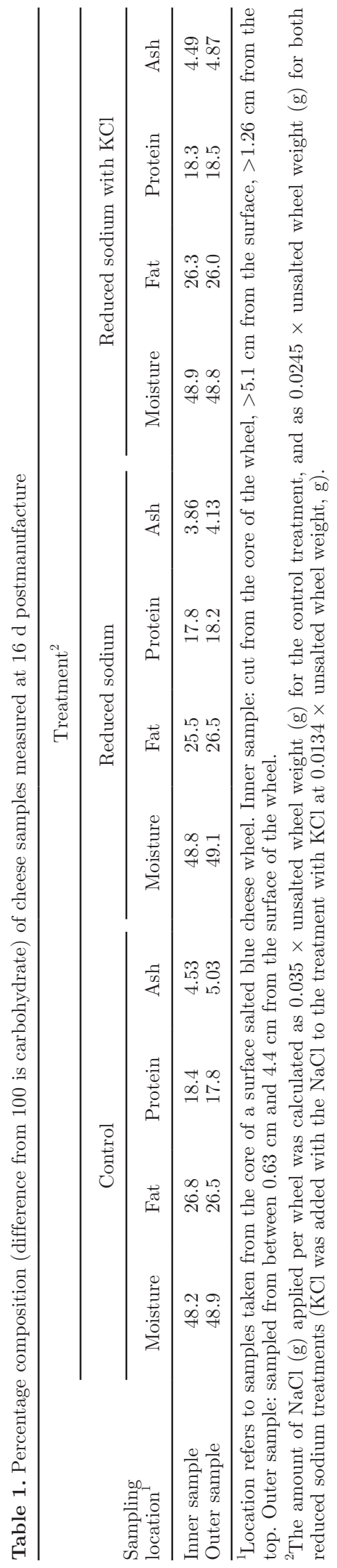

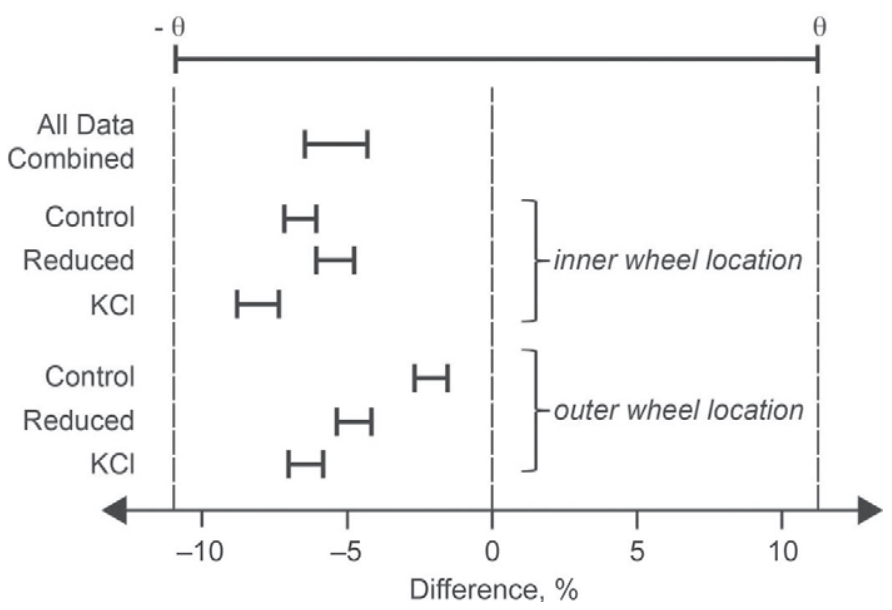

Figure 2. Percentage difference in sodium measured by atomic absorption spectrometry after microwave-accelerated digestion from that measured by dry ashing before atomic absorption; $\theta$ indicates the acceptable percentage difference from the dry ashing procedure $(11.65 \%)$. Individual treatments by location and all data (all treatments and locations combined) are indicated as $90 \%$ confidence intervals of the mean difference. The amount of $\mathrm{NaCl}(\mathrm{g})$ applied per wheel was calculated as $0.035 \times$ unsalted wheel weight $(\mathrm{g})$ for the control treatment, and as $0.0245 \times$ unsalted wheel weight $(\mathrm{g})$ for both reduced sodium treatments (reduced and $\mathrm{KCl} ; \mathrm{KCl}$ was added with $\mathrm{NaCl}$ to the $\mathrm{KCl}$ treatment at $0.0134 \times$ unsalted wheel weight, $\mathrm{g}$ ).

unsalted wheel weight $(\mathrm{g})$ for the control, and as 0.0245 $\times$ unsalted wheel weight $(\mathrm{g})$ for both reduced sodium treatments $(\mathrm{KCl}$ was added with the $\mathrm{NaCl}$ to the treatment with $\mathrm{KCl}$ at $0.0134 \times$ unsalted wheel weight, g). At each sampling time point, 2 samples of cheese were removed from both the inner and outer portions of the wheel. The inner sample was cut from the core of the wheel $>5.1 \mathrm{~cm}$ from the side and $>1.26 \mathrm{~cm}$ from the top. The outer sample was collected from a zone 0.63 $\mathrm{cm}$ from the exterior surface to $4.4 \mathrm{~cm}$ below the surface. Cheese was dry ashed in porcelain crucibles and both crucibles and cheese were prepared according to the gravimetric ash method (Wehr and Frank, 2004; method 15.041) with the following modifications. Three to five grams of cheese was weighed into the crucibles. After ashing in the muffle furnace, samples were neither rewetted nor re-ashed. The ashed sample was dissolved in nitric acid and transferred to volumetric flasks and further diluted as per method 8070:2007(E) (IDF-ISO, 2007). The source of the parameters we used for MAD was the equipment manufacturer and their recommendation for food products (CEM Corp.). All of the digestions resulted in a clear yellow fluid that appeared to be fully digested. Approximately $0.5 \mathrm{~g}( \pm 0.02 \mathrm{~g})$ of cheese was weighed and carefully placed into a PTFE reaction vessel (vessels with $100-\mathrm{mL}$ capacity and able to withstand $2,413 \mathrm{kPa}$ ), and $10 \mathrm{~mL}$ of concentrated nitric acid (68-70\% strength) was added. Tubes were closed tightly and placed into the 24-place carousel 
Table 2. Percentage difference of mean sodium measurements for samples digested by microwave from samples digested by dry ashing

\begin{tabular}{|c|c|c|c|}
\hline \multirow[b]{2}{*}{ Sampling location ${ }^{1}$} & \multicolumn{3}{|c|}{ Treatment $^{2}$} \\
\hline & Control & Reduced sodium & $\begin{array}{c}\text { Reduced sodium } \\
\text { with } \mathrm{KCl}\end{array}$ \\
\hline \multicolumn{4}{|l|}{ Inner sample } \\
\hline d 16 & $-6.1(3)$ & $-14.3(3)$ & $-9.3(3)$ \\
\hline d 31 & $-14.1(4)$ & $-6.3(4)$ & $-11.1(4)$ \\
\hline d 61 & $-8.4(4)$ & $-4.45(4)$ & $-12.4(4)$ \\
\hline d 92 & $-7.3(4)$ & $-6.0(4)$ & $-6.9(4)$ \\
\hline d 122 & $-1.05(4)$ & $-2.0(4)$ & $-5.7(4)$ \\
\hline d 153 & $-2.8(4)$ & $-0.4(4)$ & $4.2(4)$ \\
\hline Mean difference (\%) & -6.6 & -5.6 & -8.3 \\
\hline \multicolumn{4}{|l|}{ Outer sample } \\
\hline d 16 & $-4.5(3)$ & $-5.9(3)$ & $-6.8(3)$ \\
\hline d 31 & $-1.2(4)$ & $-2.9(4)$ & $-9.9(3)$ \\
\hline d 61 & $7.6(4)$ & $-4.9(4)$ & $-8.8(4)$ \\
\hline d 92 & $4.9(4)$ & $-5.2(4)$ & $-5.0(4)$ \\
\hline d 122 & $-0.3(4)$ & $-4.9(4)$ & $-4.7(4)$ \\
\hline d 153 & $7.8(4)$ & $-5.4(4)$ & $-4.3(4)$ \\
\hline Mean difference (\%) & 2.4 & -4.9 & -6.6 \\
\hline
\end{tabular}

${ }^{1}$ Location refers to samples taken from the core of a surface salted blue cheese wheel. Inner sample: cut from the core of the wheel, $>5.1 \mathrm{~cm}$ from the surface, $>1.26 \mathrm{~cm}$ from the top. Outer sample: sampled from between 0.63 and $4.4 \mathrm{~cm}$ from the surface of the wheel. Sample size in parentheses represents number of means, which are based on duplicate analyses.

${ }^{2}$ The amount of $\mathrm{NaCl}(\mathrm{g})$ applied per wheel was calculated as $0.035 \times$ unsalted wheel weight $(\mathrm{g})$ for the control treatment, and as $0.0245 \times$ unsalted wheel weight $(\mathrm{g})$ for both reduced sodium treatments $(\mathrm{KCl}$ was added with the $\mathrm{NaCl}$ to the treatment with $\mathrm{KCl}$ at $0.0134 \times$ unsalted wheel weight, g).

of the digester (MARS6 Microwave Reaction System, CEM Corp.). The digestion carousel rotated during the period of digestion and the temperature of each tube was monitored by an infrared thermometer as the tube passed over the reading zone. The microwave power was then automatically adjusted so that the tubes averaged to the targeted temperature. The temperature profile was to increase linearly over a 20 -min period to $210^{\circ} \mathrm{C}$. The samples were held at $210^{\circ} \mathrm{C}$ for $15 \mathrm{~min}$ while continuously rotating and being monitored. The microwave power required to achieve and maintain the temperature ranged from 980 to $1,060 \mathrm{~W}$. The instrument automatically adjusted the power to maintain the average working temperature among the tubes.

After tubes had cooled, each sample was transferred to a 50-mL volumetric flask and brought to volume with $0.1 N$ nitric acid before further dilution for spectroscopy. Samples from each digestion procedure were serially diluted with $0.1 \mathrm{~N}$ nitric acid to be in the linear range of calibration of the AAS and were analyzed (PerkinElmer AAnalyst 100, PerkinElmer, Waltham, MA). Results were analyzed to determine the equivalency of using MAD compared with dry ashing by using a 2 one-sided test (TOST) procedure of the mean differences between the methods, as described by Borman et al. (2009). The pooled difference between means and standard deviations of the 5 mo of data, by location and treatment, were used to obtain the up- per and lower $90 \%$ confidence intervals. Theta $(\theta)$, the predefined acceptance criterion, was calculated as the percentage difference that was 2 times the standard deviation of reproducibility $\left(\mathbf{S D}_{\mathrm{R}}\right)$ for sodium in freezedried cheese $\left(\mathrm{SD}_{\mathrm{R}}=70 \mathrm{mg} / 100 \mathrm{~g}\right)$ reported in method 119.2007(E) (ISO-IDF, 2007), from the mean of the dry-ashed control sodium cheese samples (pooled location) in our study. Linear regression was also performed on the results using mean values of each treatment at each time point. The slope of the line and intercept were compared to determine differences between the 2 measurements methods caused by cheese age and sodium migration (GraphPad Prism 6.0b for Macintosh OS X, GraphPad Software, La Jolla, CA).

Over the 5 mo of aging, sodium migrated from the outside of the cheese wheel to the interior (Figure 1). Linear regression to determine line equations followed by analysis of covariance (ANCOVA) found no significant differences between the slope of the lines for any treatment, so cheese treatment and age did not appear to affect digestion. With the exception of the outer wheel location of the control cheese, intercepts of the lines were different, with the samples digested by MAD being lower. Whether or not this bias means the results are not equivalent can by determined by using the TOST approach. This approach uses what is known about the accuracy of the original method to determine the percentage difference $(\theta)$ that could 
be observed by the new method yet still be equivalent (Chambers et al., 2005; Chatfield and Borman, 2009). We chose to use the data in the IDF method for determination of sodium in dairy products by AAS for the historical data (ISO-IDF, 2007). In that method, the authors list the results of their interlaboratory trials for a freeze-dried cheese sample with a mean value of $1,680 \mathrm{mg}$ of sodium/100 $\mathrm{g}$ and an $\mathrm{SD}_{\mathrm{R}}$ of $70 \mathrm{mg} / 100 \mathrm{~g}$. Using 2 times this standard deviation, we determined $\theta$ to be $11.65 \%$. Table 2 shows the percentage difference of the mean sodium values quantified from samples that were digested by MAD from those dry ashed. As shown in Figure 2, confidence intervals of the mean differences for all data combined and for each treatment individually all fell within the acceptable percentage difference. An alternative way to assess the similarity of the measurements produced by the 2 digestion methods is to consider the reproducibility limit stated in the IDF sodium method (ISO-IDF, 2007). They state that the absolute differences between laboratories using the same sample and method on different equipment to measure sodium could not exceed $19 \%$ in more than $5 \%$ of the cases. In the current study, the largest measurement difference was $14.3 \%$. For the majority of data in our study, the measurement difference was well below $10 \%$. Irrespective of the approach used to determine acceptable limits of method equivalence, our study indicates that the MAD method described can be used as a replacement to dry ashing of blue cheese for sodium analysis before AAS.

\section{REFERENCES}

AOAC International. 2011. Official Method 2011.14: Calcium, copper iron, magnesium, manganese, potassium, phosphorus, sodium and zinc in fortified food products. Microwave digestion and inductively coupled plasma-optical emission spectrometry. AOAC International, Gaithersburg, MD.

Borman, P. J., M. J. Chatfield, I. Damjanov, and P. Jackson. 2009. Design and analysis of method equivalence studies. Anal. Chem. 81:9849-9857.

Chambers, D., G. Kelly, G. Limentani, A. Lister, K. R. Lung, and E. Warner. 2005. Analytical method equivalency: An acceptable analytical practice. Pharmaceut. Technol. 29:64-80.

Chatfield, M. J., and P. J. Borman. 2009. Acceptance criteria for method equivalency assessments. Anal. Chem. 81:9841-9848.

ISO-IDF (International Organization for Standardization and International Dairy Federation). 2007. Milk and milk products: Determination of calcium, sodium, potassium and magnesium contents-Atomic absorption spectrometric method. IDF119:2007/ ISO 8070:2007. ISO, Geneva, Switzerland; IDF, Brussels, Belgium.

Julshamn, K., P. Lea, and H. S. Norli. 2005. Determination of sodium in foods by flame atomic absorption spectrometry after microwave digestion: NMKL Interlaboratory study. J. AOAC Int. 88:12121216.

Wehr, H. M., and J. F. Frank. 2004. Standard Methods for the Examination of Dairy Products. 17th ed. American Public Health Association, Washington, DC. 